\title{
Dissection of Extracranial Internal Carotid Artery Due to Balloon Guiding Catheter Resulting in Asymptomatic Internal Carotid Artery Occlusion
}

\author{
Suha Akpinar, ${ }^{1}$ and Guliz Yilmaz ${ }^{2,}$ \\ ${ }^{1}$ Near East University Hospital, Nicosia, North Cyprus, Turkey \\ ${ }^{2}$ Department of Radiology, Faculty of Medicine, Near East University Hospital, Nicosia, North Cyprus, Turkey \\ 'Corresponding author: Guliz Yilmaz, Department of Radiology, Faculty of Medicine, Near East University Hospital, Nicosia, North Cyprus, Turkey. Tel: +90-3926751000; \\ +90-5338748687, Fax: +90-3926751090, E-mail: glz.yilmaz@hotmail.com
}

Received 2015 February 23; Revised 2015 April 12; Accepted 2015 June 22.

\begin{abstract}
Dissection of the internal carotid artery (ICA) is a rare condition that accounts for a significant proportion of ischemic strokes in young adults. Iatrogenic dissection as a complication of neurointerventional procedures is a traumatic dissection which has been reported relatively rare in the literature. In this report, a case of dissection of the ICA is reported that was caused by repetitive movement of the balloon guiding catheter during stent-assisted thrombectomy (SAT), resulting in occlusion of the ICA.
\end{abstract}

Keywords: Dissection, Internal Carotid Artery, Neurointerventional Procedure, Magnetic Resonance Angiography

\section{Introduction}

Internal carotid artery (ICA) dissections may occur spontaneously or following trauma, whereas extracranial carotid artery dissections are rare conditions that account for a significant proportion of ischemic strokes in young adults (1). On the other hand, iatrogenic dissection is a kind of traumatic dissection that may occur as a complication of neurointerventional procedures (2-4). The interventionalist has to decide whether to continue with the intracranial procedure or manage the dissection by either using antithrombotics or endovascular management during or after the intervention (5). Current paper reports a dissection of the extracranial segment of the ICA in a stroke case which occurred as a result of repetitive movement of the balloon guiding catheter during stent-assisted thrombectomy (SAT) that progressed to late occlusion days after the dissection.

\section{Case Presentation}

A 66-year-old woman was brought to the emergency department with persistent symptoms of right-sided hemiparesis and aphasia. She had the same complaints twice within six hours, but not lasting more than five minutes. She was examined by cranial magnetic resonance imaging (MRI), including magnetic resonance angiography (MRA), axial fluid attenuated inversion recovery (FLAIR) images, susceptibility weighted images (SWI), and diffusion weighted images (DWI); these revealed a patchy, restricted diffusion in the left hippocampus and left occipital lobe. MRA showed a thrombus occluding the ophthalmic segment of the ICA extending to the supraclinoid segment (Figure $1 \mathrm{~A}$ ). Under treatment with heparin, she was examined by MRA 24 hours later due to recurrence of her symptoms. It showed a new diffusion restriction in the territory of the middle cerebral artery (MCA) with total occlusion of the left MCA in M1 segment. There was only restriction on DWI without any signal change on FLAIR sequences, which were compatible with hyperacute ischemia. As the stroke symptoms were within the first six hours, without established findings of infarction on MRI, she was admitted for emergent SAT, which has to be performed within the critical time window in the anterior circulation of six hours for intraarterial thrombolysis and eight hours for SAT.

Under general anesthesia, an $8 \mathrm{~F}$ balloon guiding catheter (Corail, Balt, France) was placed at the distal part of the left ICA cervical segment in order to decrease the migration of the dislodged thrombus. The occlusion site was reached with Transend 14 microwire(Stryker, USA) and Rebar $2.8 \mathrm{~mm}$ microcatheter (Covidien, USA) and SAT was performed with $5 \times 30 \mathrm{~mm}$ Solitaire (Covidien, USA) stent three times (Figure $1 \mathrm{~B}$ ). Focal dissection of the ICA at the distal cervical segment was noticed due to the repeated to and fro movement of the inflated balloon guiding catheter during the thrombectomy procedure, although the balloon was inflated and deflated during every SAT pass. 

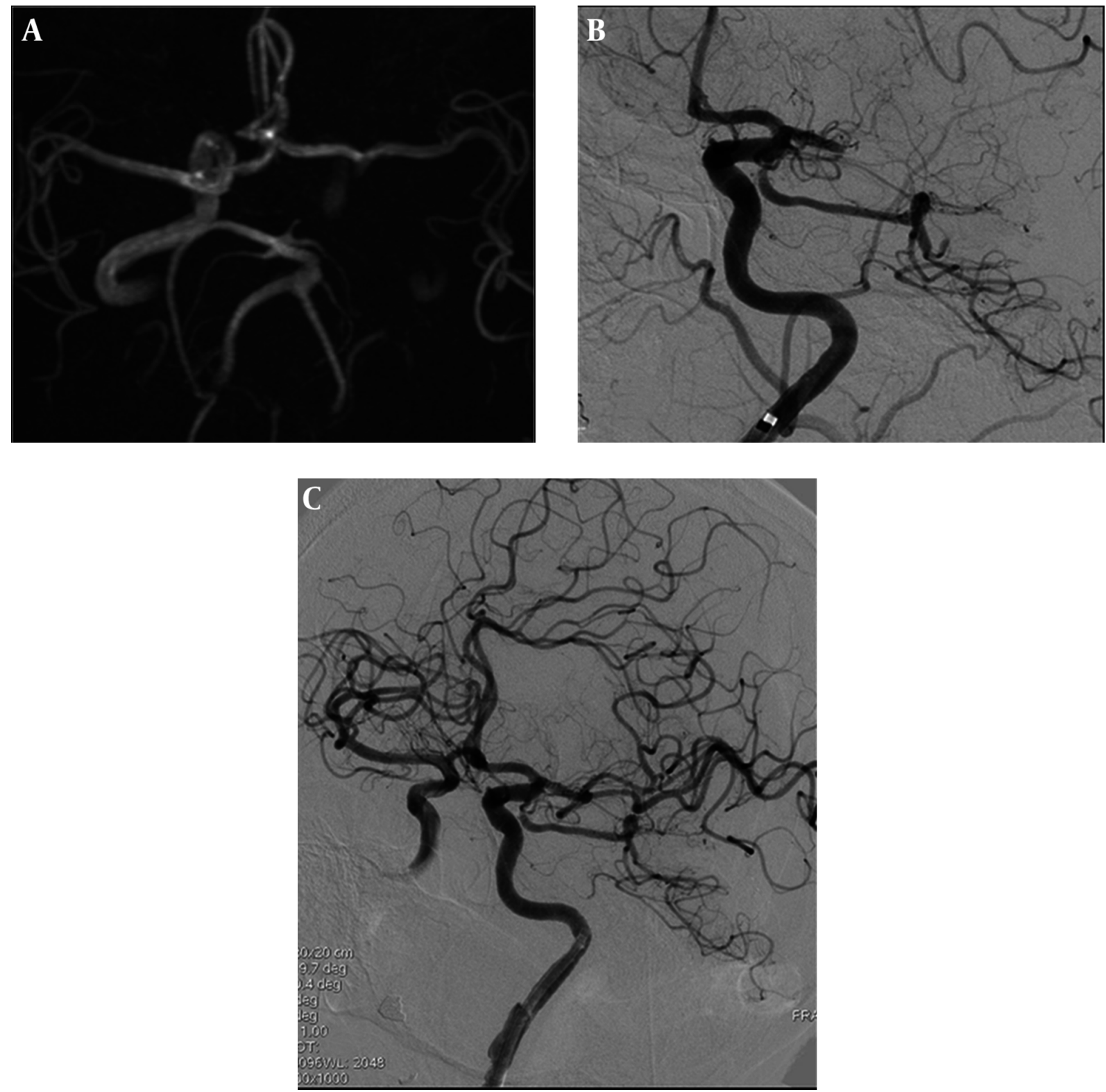

Figure 1. A 66-year-old woman with middle cerebral artery thrombosis underwent stent-assisted thrombectomy. A, Cranial axial reformatted MRA performed at the time of admission showed a thrombus occluding the left ICA extending to the supraclinoid segment. Left MCA flow was from the anterior communicating artery; B, Cranial digital subtraction angiography demonstrated the guiding catheter at the distal part of the left ICA cervical segment and the occlusion of the left MCA; C, Cranial digital subtraction angiography showed a focal, nonprogressive ICA dissection at the distal cervical segment and recanalization of the MCA. Note the patient's left ICA segment.

For anterior circulation strokes, while withdrawing the thrombus with the aid of the thrombectomy stent, there is a risk of dislodgment of the thrombus to distal branches of the MCA and anterior cerebral artery (ACA). In order to decrease the risk of dislodgment, it is preferred to use a balloon guiding catheter to stop the flow and perform suction during withdrawal of the thrombus.

The dissection did not cause any flow disturbance or progression at the left ICA and the MCA was recanalized after the fourth SAT (Figure $1 \mathrm{C}$ ). Hence, the procedure was finalized without stenting. The patient had no additional symptoms and her clinical status progressively improved over the following days. The patient was placed on a low dose of heparin during the follow-up period, which is the accepted follow-up guideline for dissections and she was coumadinized on the seventh day. The etiology of the 
stroke was accepted as being of cardioembolic origin because she was diagnosed as having atrial fibrillation on electrocardiography.

There was no pathological signal on MRI after the stroke treatment and her 24-hour control computed tomography. On the seventh day before discharge, the left ICA lumen was almost occluded on a color Doppler ultrasonography scan. Although the patient was on coumadin treatment, the dissection flap at the cervical ICA segment had progressed to the ophthalmic segment distally, downwards to the ICA orifice, and was occluded on MRA. The patient was asymptomatic at discharge and a follow-up scan six to eight weeks later was planned. She did not have any complaints during the eight weeks, but the dissected ICA segment did not recanalize and the occlusion extended from the ICA orifice to the ophthalmic segment of the ICA based on MRA, despite being on coumadin therapy (Figure 2).

\section{Discussion}

Iatrogenic dissection as a complication of neurointerventional procedures usually occurs when there is a tear in the intima of the arterial wall leading to intrusion of blood within the layers and formation of a false lumen. A false lumen often causes flow limitation to a variable extent $(1$, $5,6)$. Paramasivam et al. reported an incidence of $0.25 \%$ and Akins et al. reported cervical carotid artery dissection in five cases of 144 (3.5\%) during endovascular treatment for acute stroke $(7,8)$. None of these studies reported the complications in relation with the catheter type except for Akins et al. They reported the incidence of dissection to be $4.5 \%$ using the Solitaire device which was the thrombectomy stent used in the present case (8).

As mentioned before, there are very few cases in the literature, because some operators may consider small and asymptomatic dissections to be "technical events", rather than complications, and therefore not report them (5).

Although it has not been statistically proven that iatrogenic dissections occur more frequently during neurointerventional procedures than during diagnostic cerebral angiography, the risk of iatrogenic dissection may be slightly elevated in neurointerventional procedures due to an increased number of vascular runs, catheter exchanges, and distal placement of catheters (5). Paramasivam et al. argued that most cases had multiple manipulations, ranging from one to six, in the dissected vessels (7). In this study, it was noted that the dissection of the extracranial ICA after three SAT passes during the stroke treatment was due to the repetitive movement of the balloon guiding catheter, which was not identified in a search of literature.

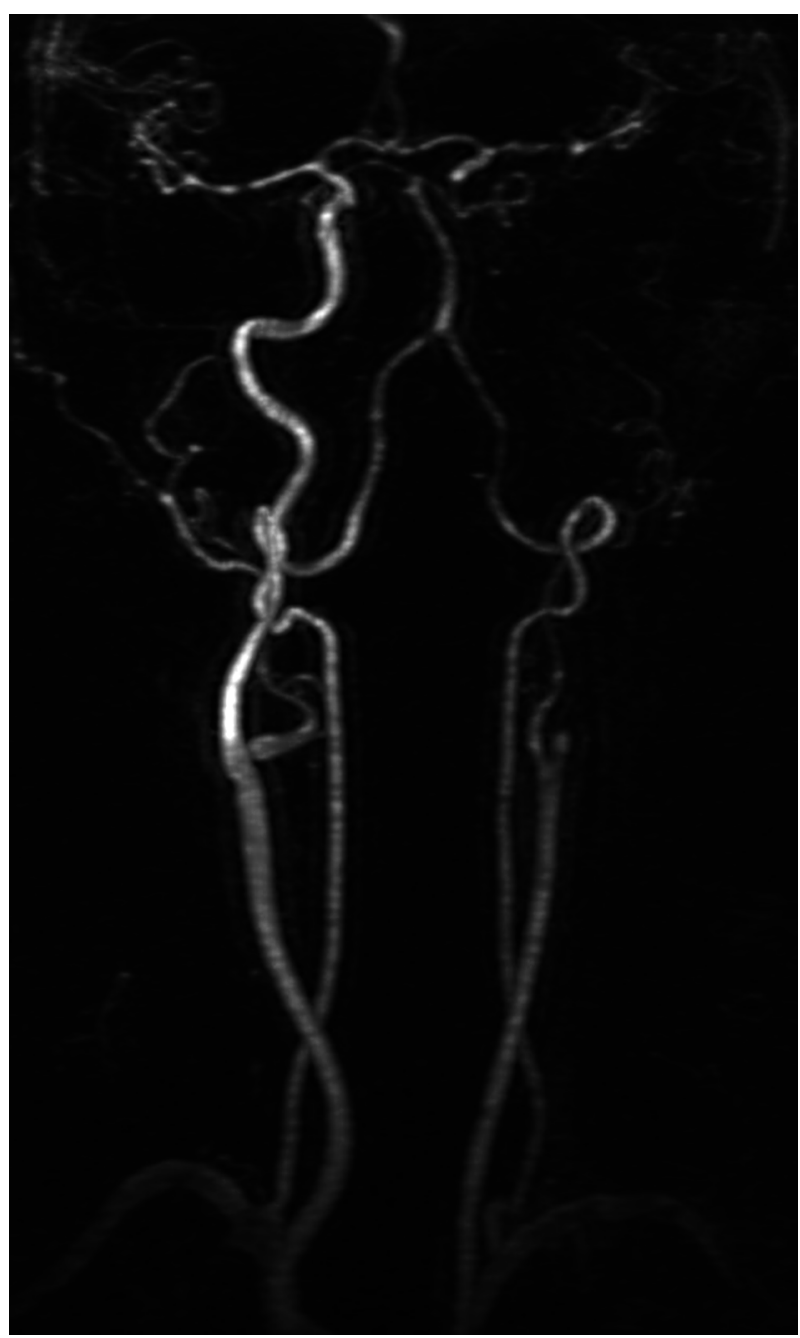

Figure 2. Contrast-enhanced coronal reformatted craniocervical MRA demonstrated the occluded left ICA extending from the proximal segment to the supraclinoid segment.

Although there is no specific therapy for dissection of the ICA, the most common application for symptomatic spontaneous or traumatic dissections is intravenous administration of heparin. Hart and Easton recommend a follow-up protocol which consists of using heparin for seven days and coumadin for three months. Antiplatelet agents are recommended only when coumadin is contraindicated (9). If there is no sign of ischemia, aspirin may be recommended for dissections $(5,9)$. Good outcomes have been reported with this treatment (6). There are reports that suggest stenting as an optional treatment, whereas Paramasivam et al. stented cases with luminal stenosis of over 70\% and poor intracranial circulation (7). Stroke resulting from dissection usually occurs within the 
first week, however it may occur after up to a one month period. For this reason, it is suggested to administer anticoagulant therapy for at least one month (10).

In this case, the intervention was continued without treating the proximal dissection as there was no progression of the dissection to cause any flow disturbance or poor intracranial circulation.

A dissection of the ICA with a balloon guiding catheter and its progression to the proximal segment of the vessel is a rare condition. Although the dissection is not usually progressive and does not usually cause stenosis, silent occlusion may be the outcome in the dissected segment. For this reason, anticoagulant therapy and follow-up imaging are necessary for nonprogressive iatrogenic dissections following neurointerventional procedures.

\section{Footnotes}

Authors' Contribution: Study concept and design: Suha Akpinar; analysis and interpretation of data: Suha Akpinar and Guliz Yilmaz; drafting of the manuscript: Suha Akpinar and Guliz Yilmaz; critical revision of the manuscript for important intellectual content: Suha Akpinar and Guliz Yilmaz.

Financial Disclosure: The authors declare that they have nothing to disclose.

\section{References}

1. Schievink WI. Spontaneous dissection of the carotid and vertebral arteries. $N$ Engl J Med. 2001;344(12):898-906. doi: 10.1056/NEJM200103223441206. [PubMed:11259724].

2. Ansari SA, Thompson BG, Gemmete JJ, Gandhi D. Endovascular treatment of distal cervical and intracranial dissections with the neuroform stent. Neurosurgery. 2008;62(3):636-46. doi: 10.1227/01.NEU.0000311350.25281.6B. [PubMed: 18301346].

3. Donas KP, Mayer D, Guber I, Baumgartner R, Genoni M, Lachat M. Endovascular repair of extracranial carotid artery dissection: current status and level of evidence. J Vasc Interv Radiol. 2008;19(12):1693-8. doi:10.1016/j.jvir.2008.08.025. [PubMed: 18845451].

4. Menon R, Kerry S, Norris JW, Markus HS. Treatment of cervical artery dissection: a systematic review and meta-analysis. I Neurol Neurosurg Psychiatry. 2008;79(10):1122-7. doi: 10.1136/jnnp.2007.138800. [PubMed: 18303104].

5. Cloft HJ, Jensen ME, Kallmes DF, Dion JE. Arterial dissections complicating cerebral angiography and cerebrovascular interventions. AJNR Am J Neuroradiol. 2000;21(3):541-5. [PubMed: 10730648].

6. Anson J, Crowell RM. Cervicocranial arterial dissection. Neurosurg. 1991;29(1):89-96.

7. Paramasivam S, Leesch W, Fifi J, Ortiz R, Niimi Y, Berenstein A Iatrogenic dissection during neurointerventional procedures: a retrospective analysis. J Neurointerv Surg. 2012;4(5):331-5. doi: 10.1136/neurintsurg-2011-010103. [PubMed: 21990537].

8. Akins PT, Amar AP, Pakbaz RS, Fields JD, Swift Investigators. Complications of endovascular treatment for acute stroke in the SWIFT trial with solitaire and Merci devices. AJNR Am J Neuroradiol. 2014;35(3):524-8. doi: 10.3174/ajnr.A3707. [PubMed: 24029392].

9. Hart RG, Easton JD. Dissections of cervical and cerebral arteries. Neurol Clin. 1983;1(1):155-82. [PubMed: 6680158].

10. Biousse V, D'Anglejan-Chatillon J, Touboul PJ, Amarenco P, Bousser MG. Time course of symptoms in extracranial carotid artery dissections. A series of 80 patients. Stroke. 1995;26(2):235-9. [PubMed: 7831694]. 